\title{
Optimal Road Toll Design from the Perspective of Sustainable Development
}

\author{
Lin Cheng and Fei Han \\ School of Transportation, Southeast University, Nanjing, Jiangsu 210096, China \\ Correspondence should be addressed to Fei Han; 230129437@seu.edu.cn
}

Received 5 July 2014; Accepted 23 August 2014; Published 1 September 2014

Academic Editor: Yongjun Shen

Copyright (C) 2014 L. Cheng and F. Han. This is an open access article distributed under the Creative Commons Attribution License, which permits unrestricted use, distribution, and reproduction in any medium, provided the original work is properly cited.

\begin{abstract}
This paper investigates the optimal road toll design problem from the perspective of sustainable development at network-wide level. In this paper the sustainable development level of transportation system is quantitatively described with the total vehicular emission, total fuel consumption, and total travel time in the network. In order to simultaneously consider the impacts of all these three indicators on sustainability of transportation system, we integrate them into a sustainable development index (SD-index) by a linear combination, and then we establish the corresponding bilevel optimization model. The upper level problem is the network toll design problem to maximize the SD-index from the viewpoint of traffic managers, and the lower level problem is to depict travelers' route choice behavior under a certain road toll scheme. Finally, a combined genetic algorithm and gradient projection algorithm (GA-GP) is used to solve the bilevel model, in which the GP algorithm solves the traffic assignment problem with road toll scheme in the lower level. In order to verify the proposed model and algorithm, we take the Nguyen-Dupuis network for the numerical example, and the computing results show that the model and algorithm are effective and efficient.
\end{abstract}

\section{Introduction}

Traffic congestion is a global problem that almost everyone living in big cities has to face, which causes a great waste of time for the urban commuters. At the same time, the rapid development of transportation, especially the current unrestrictive use of private cars, also brings about the severe air pollution and energy crisis problem. It is well known that all these traffic problems seriously restrict the further development for the cities all over the world. And therefore, there is clearly an urgent need for effective measures and policies to combat all these issues caused by the unsustainable transportation systems and then to develop a sustainable, low-carbon, and energy-saving urban transportation systems.

Road toll pricing, which is a type of traffic demand management measure, is widely recognized as a useful tool for alleviating traffic congestion and reducing vehicular emissions [1-6]. Recent developments in information and communication technologies have made the implementation of road pricing schemes easier. There are some wellknown successful examples of electronic road pricing all over the world, including the congestion charging schemes in California, Singapore, and London $[7,8]$.

There is a substantial body of literatures on road toll pricing, and the policy objectives of these works can be generally summarized as follows: minimizing total travel time of transportation networks with fixed travel demand [1-3, $9,10]$, maximizing total social welfare of all travelers in the network with elastic travel demand [11-14], maximizing the network capacity [15], or maximizing the network travel time reliability [16], and so on. For recent reviews on road tolls, see $[17,18]$. However, as the issues of society, environment, and energy caused by traffic congestion become worse and worse, improving the sustainability of the urban transportation systems is nowadays the urgent concern of transportation network management. In this paper, we attempt to investigate the optimal road toll design problem from the perspective of sustainable development of transportation system. In order to quantitatively measure the sustainability of transportation system with different network toll schemes, some quantitative indicators must be adopted. In general, the sustainable development level of transportation system can be described from the aspects of transportation network efficiency and 
environmental pollution, as well as the energy consumption. Consequently, in this paper we take the network-wide total travel time, total vehicular emission, and total fuel consumption as the measuring indicators of the sustainable development level of transportation system, and furthermore, in order to simultaneously consider the impacts of all these three indicators on the sustainability of transportation system, we integrate all these three sustainability indicators into a sustainable development index (SD-index) by a linear combination. And thus, the optimization objective in the road toll design problem is to maximize the SD-index of the urban transportation system.

The remaining sections are organized as follows. Section 2 presents the bilevel programming model of optimal road toll design problem from the perspective of sustainable development. Section 3 introduces the solution approach, and in this section we adopt a combined GA-GP algorithm to solve the bilevel optimization model. In order to verify the proposed model and algorithm, the Nguyen-Dupuis network is taken as a numerical example in Section 4 and conclusions are provided in Section 5.

\section{Bilevel Programming Model}

Since the sustainable development index (SD-index) is determined by the total weighted sum of the emitted vehicular emission and the fuel consumption, as well as the total travel time in the network, and, furthermore, these three sustainability indicators can be largely affected by the link flows, thus we must figure out the relationships between these sustainability indicators and link flows before getting into the essential investigation; in other words, we must figure out how these sustainability indicators change with respect to the link flow volumes in the network. In fact, there are many investigations having focused on deriving the desirable function relationships to date, and some analytical function expressions are obtained based on the realistic survey data $[4,5,19,20]$. In this paper, we adopted the emission function used in TRANSYT-7F $[4,5,19]$ and the fuel consumption function derived in [20], as well as the commonly used BPR function.

The optimal road toll design problem at network-wide level from the perspective of sustainable development with fixed demand static user equilibrium flow constraint is formulated as follows:

$$
\begin{aligned}
\max I(\mathbf{x}, \boldsymbol{\tau})=\exp ( & \left.-\frac{Z(\mathbf{x}, \boldsymbol{\tau})}{10^{m}}\right) \\
Z(\mathbf{x}, \boldsymbol{\tau})=\sum_{a}[ & \alpha t_{a}\left(x_{a}(\boldsymbol{\tau}), \tau_{a}\right) x_{a}(\boldsymbol{\tau}) \\
& +\beta E_{a}\left(x_{a}(\boldsymbol{\tau}), \tau_{a}\right) x_{a}(\boldsymbol{\tau}) \\
& \left.+\gamma F_{a}\left(x_{a}(\boldsymbol{\tau}), \tau_{a}\right) * \frac{l_{a}}{100} x_{a}(\boldsymbol{\tau})\right] \\
E_{a}\left(x_{a}(\boldsymbol{\tau}), \tau_{a}\right)= & 0.2038 t_{a}\left(x_{a}(\boldsymbol{\tau}), \tau_{a}\right) \\
& \times \exp \left(0.7962 \frac{l_{a}}{t_{a}\left(x_{a}(\boldsymbol{\tau}), \tau_{a}\right)}\right)
\end{aligned}
$$

$$
\begin{aligned}
F_{a}\left(x_{a}(\boldsymbol{\tau}), \tau_{a}\right)= & 0.0021\left[\frac{l_{a}}{t_{a}\left(x_{a}(\boldsymbol{\tau}), \tau_{a}\right)}\right]^{2} \\
& -0.3465 \frac{l_{a}}{t_{a}\left(x_{a}(\boldsymbol{\tau}), \tau_{a}\right)}+26.135
\end{aligned}
$$$$
\text { s.t. } \quad 0 \leq \tau_{a} \leq U_{a} \quad \forall a \in A \text {, }
$$

where $\mathbf{x}(\boldsymbol{\tau})$ is the equilibrium flow pattern defined by the following fixed demand static user equilibrium problem with a certain road toll scheme imposed on the network:

$$
\begin{array}{ll}
\min & z(\mathbf{x})=\sum_{a} \int_{0}^{x_{a}}\left[t_{a}\left(\omega, \tau_{a}\right)+\frac{\tau_{a}}{\rho}\right] d \omega \\
\text { s.t. } & \sum_{k} f_{k}^{r s}=q^{r s} \quad \forall r \in \mathbf{R}, s \in \mathbf{S} \\
& x_{a}=\sum_{r s} \sum_{k \in K^{r s}} \delta_{a, k}^{r s} f_{k}^{r s} \quad \forall r \in \mathbf{R}, s \in \mathbf{S}, a \in \mathbf{A}, k \in \mathbf{K}^{r s} \\
& f_{k}^{r s} \geq 0 \quad \forall r \in \mathbf{R}, s \in \mathbf{S}, k \in \mathbf{K}^{r s} .
\end{array}
$$

The notations are defined in Notations section.

In this bilevel model, the upper level describes leader or policy problem and the lower-level model represents follower or user's behavioral problem. In the optimal road toll design problem from the perspective of sustainable development, the upper-level problem is to determine an optimal decision for road toll scheme to make the sustainable development index (i.e., SD-index) maximum. The lower-level problem represents a user equilibrium assignment problem that describes travelers' path-choosing behavior under a certain road toll scheme.

The proposed bilevel model is generally difficult to solve because evaluation of the upper-level objective functions requires solving the lower-level subprogram. Besides, since this bilevel model is a NP-hard problem and the upper level is nonconvex and nondifferentiable in $\mathbf{x}$ which is defined by the lower level model, therefore, the existing exact solution algorithms are not available for solving this bilevel model. Consequently, in this paper we adopt a combined genetic algorithm and gradient projection algorithm (GAGP) to solve the proposed model; to be specific, the traffic assignment problem with road toll in the lower level is solved by GP algorithm while the toll design problem in the upper level is solved by GA.

\section{Solution Approach}

Genetic algorithm (GA) is one of the most well-known search heuristics for solving optimization problems [21-24]. GA uses techniques such as inheritance, mutation, selection, and crossover which are inspired by natural evolution and can search for optimal or near-optimal solutions for an optimization problem over the search domain. GA is implemented as a computer simulation in which a population of chromosomes of candidate solutions to an optimization problem evolves 
toward better solutions. Solutions can be represented in binary or real-coded. The evolution usually starts from a population of randomly generated individuals and happens in generations. In each generation, the fitness value of every individual in the population is evaluated; multiple individuals are randomly selected from the current population (based on their fitness value) and modified (recombined and possibly randomly mutated) to form a new population. The new population is then used in the next iteration of the algorithm. Commonly, the algorithm terminates when either a maximum number of generations have been produced, or a satisfactory fitness level has been reached for the population.

In this paper the chromosomes of the GA are designed in this way: all the tolled links on the network are successively numbered, and each gene in one chromosome represents the toll level charged on the corresponding link. For the chromosomes in the initial generation, all their genes are randomly generated between 0 and $U$. And meanwhile, since the optimal toll design variables in the upper-level subprogram are real, we adopt the real-coded representation to represent the design variables $\tau_{a}, \forall a \in A$ with a length equal to the number of tolled links in the network. The value of each gene represents the link toll level, which is constrained by the upper and lower bound, that is, $U$ and 0 in this paper. In the following, a brief description of the GA implementation procedure is provided. The implementation procedure of GA is shown in Figure 1.

Step 1 (Initialization). A feasible search space should be first defined, and all the chromosomes will be operated and evaluated in this constrained space. Set the maximum number of generations as $T$, population size as $N$, crossover probability as $P_{c}$, and mutation probability as $P_{m}$. Then set $t=0$, and create the original population [Pop(0)] consisting of $N$ chromosomes, which can be denoted by $[\operatorname{Pop}(0)]_{1},[\operatorname{Pop}(0)]_{2}, \ldots,[\operatorname{Pop}(0)]_{i}, \ldots,[\operatorname{Pop}(0)]_{N}$. It is noteworthy that these chromosomes are randomly generated from the feasible region, the length of each chromosome equals the number of the tolled links in the network, and each gene of the chromosomes represents the corresponding link toll level which lies in the interval between 0 and $U$.

Step 2. For each chromosome $[P o p(t)]_{i}(i=1,2, \ldots, N)$ in the current population $[\operatorname{Pop}(t)]$, solve the lower level program by Gradient Projection (GP) algorithm and evaluate the fitness value with the sustainable development index (SDindex), which is the objective function of the upper level program.

Step 3. If $t>T$, then stop and output the optimal chromosome; else set $t:=t+1$, and then go to next step (Step 4).

Step 4 (Reproduction). In this process the chromosomes reproduce according to their fitness values; that is, the bigger the $\mathrm{SD}$-index value is, the higher the chance becomes that the chromosome will be chosen as the offspring chromosome. And meanwhile, a simpler tournament selection is adopted to decide whether or not a chromosome can reproduce depending on SD-index value. The procedure of tournament selection is that $p_{r} \cdot N$ chromosomes with maximum SDindex values are added into the population and $p_{r} \cdot N$ chromosomes with minimum SD-index values are discarded from the population. The resulting population has the same size with the original one. After the selection, all chromosomes are completely put in the mating pool.

Step 5 (Crossover). Since the chromosomes in the toll design problem are coded using a real-coded representation, the arithmetic crossover is used. This method randomly divides the $N$ chromosomes into $N / 2$ pairs, and each pair yields two new chromosomes via a linear combination. Assume that both $\boldsymbol{\tau}$ and $\boldsymbol{\tau}^{\prime}$ are selected and $c$ is a random number chosen from 0 to 1 . If $c>p_{c}$, then the following crossover operations for $\mathbf{y}$ and $\mathbf{y}^{\prime}$ are performed:

$$
\begin{array}{ll}
\text { If } I(\boldsymbol{\tau})>I\left(\boldsymbol{\tau}^{\prime}\right) & \\
\text { then } \overline{\boldsymbol{\tau}}=\boldsymbol{\tau}+\lambda\left(\boldsymbol{\tau}-\boldsymbol{\tau}^{\prime}\right), & \overline{\boldsymbol{\tau}}^{\prime}=\boldsymbol{\tau}^{\prime}+\lambda\left(\boldsymbol{\tau}-\boldsymbol{\tau}^{\prime}\right) ; \\
\text { Else } \overline{\boldsymbol{\tau}}=\boldsymbol{\tau}+\lambda\left(\boldsymbol{\tau}^{\prime}-\boldsymbol{\tau}\right), & \overline{\boldsymbol{\tau}}^{\prime}=\boldsymbol{\tau}^{\prime}+\lambda\left(\boldsymbol{\tau}^{\prime}-\boldsymbol{\tau}\right),
\end{array}
$$

where $I(\boldsymbol{\tau})$ and $I\left(\boldsymbol{\tau}^{\prime}\right)$ are, respectively, the fitness values of chromosomes $\boldsymbol{\tau}$ and $\boldsymbol{\tau}^{\prime}$, while $\overline{\boldsymbol{\tau}}$ and $\overline{\boldsymbol{\tau}}^{\prime}$ are the resulting children chromosomes, and $\lambda \in[0,1]$ is a random number determining the crossover grade of chromosomes $\boldsymbol{\tau}$ and $\boldsymbol{\tau}^{\prime}$. If $c<p_{c}$, no crossover operation is performed.

Step 6 (Mutation). With a lower probability, randomly choose some genes from all the chromosomes in current population, and then modify value of these genes by a pseudorandom number between 0 and $U$. In this paper the multiple mutation is adopted and only $p_{m} \cdot N$ random chromosomes in the population $[\operatorname{Pop}(\mathbf{t})]$ are chosen to be mutated. The formula of mutation operation for a selected $\tau$ is given by

$$
\boldsymbol{\tau}_{\text {new }}=\boldsymbol{\tau}+\alpha \boldsymbol{\theta},
$$

where $\alpha$ is a small positive constant and $\boldsymbol{\theta}$ is a random perturbation vector to produce small disturbances on $\boldsymbol{\tau}$. If the resulting chromosome is outside the feasible region, the original one is retained. This process may also generate some new chromosomes.

Step 7. After the genetic operations of reproduction, crossover, and mutation, a new offspring population will be generated. And then go back to Step 2.

\section{Numerical Example}

In this section, an example demonstrates the performance of the proposed model and algorithm for the optimal toll design problem. The network used in the numerical experiment is depicted in Figure 2, which was also used for numerical examples in [25]. The road network consists of 13 nodes, 19 links, and 25 paths. There are $4 \mathrm{O}-\mathrm{D}$ pairs; that is, $1 \rightarrow 2,1 \rightarrow 3$, $4 \rightarrow 2$, and $4 \rightarrow 3$. The travel demands for each OD pair are 


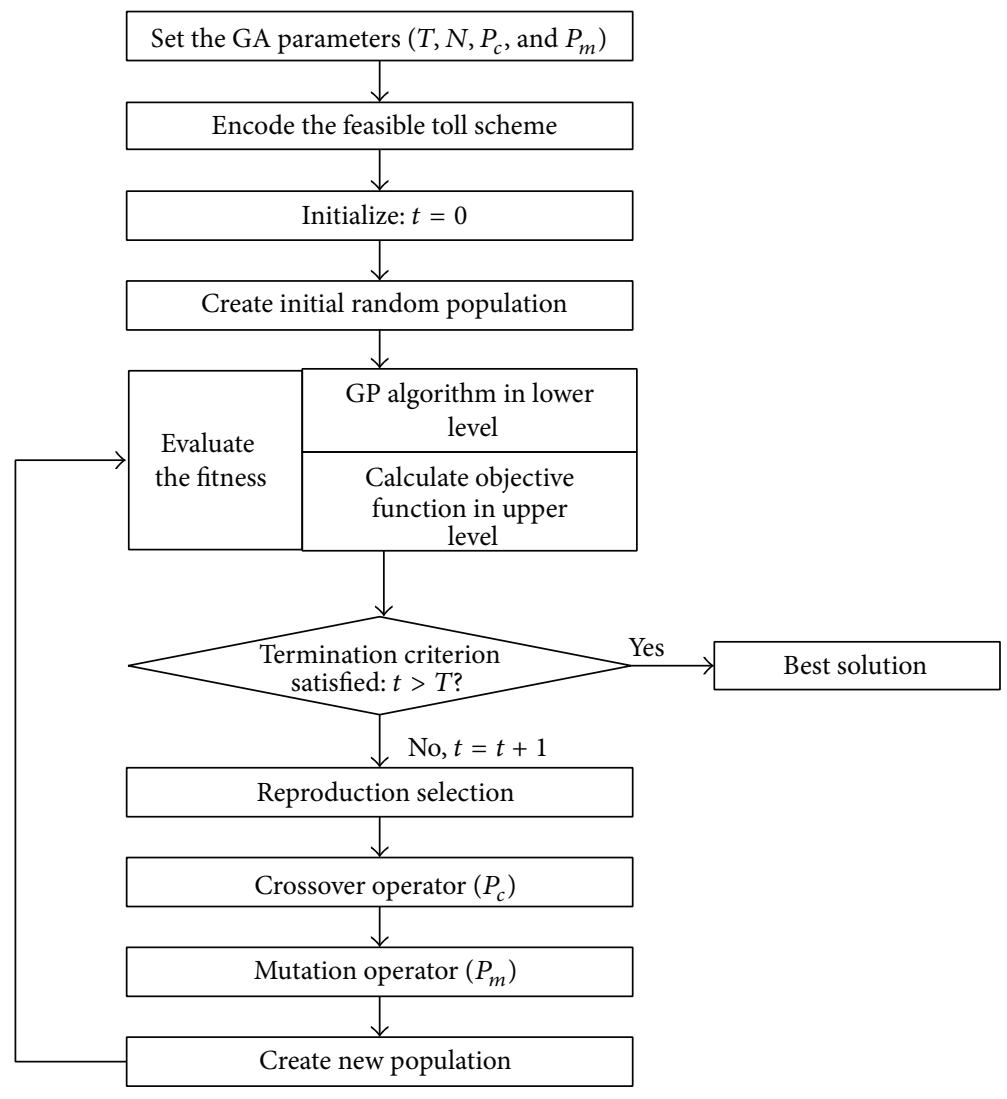

FIGURE 1: The implementation procedure of genetic algorithm.

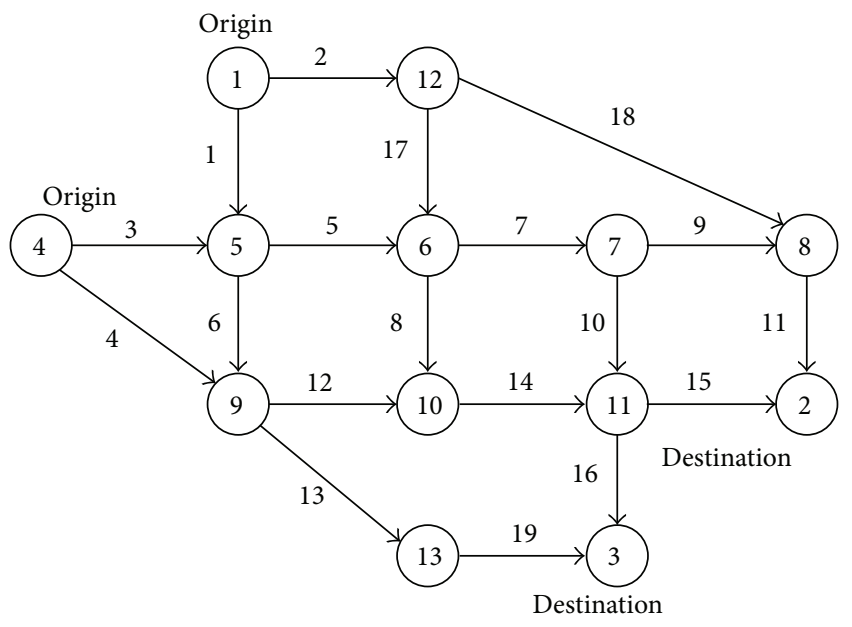

FIGURE 2: Nguyen-Dupuis network used in the example.

given in Table 1. The link travel time function is the standard BPR function:

$$
t_{a}=t_{a}^{0}\left[1+0.15\left(\frac{v_{a}}{C_{a}}\right)^{4}\right], \quad a=1,2, \ldots, 19,
$$

where $t_{a}, t_{a}^{0}, v_{a}$, and $C_{a}$ are the travel time, free-flow travel time, flow, and capacity of link $a$, respectively. All the link related parameters are provided in Table 2.
TABle 1: Travel demands of each OD pair (veh/hr).

\begin{tabular}{cccc}
\hline $\mathrm{O}$ & & $\mathrm{D}$ & \\
& 2 & 3 \\
\hline 1 & 400 & 800 \\
4 & 600 & 200 \\
\hline
\end{tabular}

In this paper, Matlab GA Toolbox is applied to solve the bilevel programming model, with the GA parameters set as follows: $T=100, N=20, P_{c}=0.65$, and $P_{m}=0.01$.

The optimal toll scheme and the equilibrium link flow pattern under UE, as well as the corresponding link travel times, are presented in Table 3. Besides, in order to compare the results of the toll scenario with the no-toll scenario and analyze the impacts of road toll on SD-index, we also provide the UE solution of no-toll scenario in Table 3. In addition, under UE with the optimal road toll scheme the used equilibrium paths between each OD pair and the corresponding path flows, as well as the resultant path costs (in fact, the path cost is generalized path travel cost which consists of the path travel time and the total toll charged on that path), are given in Table 4. And meanwhile, the used paths and the equilibrium path flows under UE without road toll are also presented in Table 4 .

As shown in Table 3, when there is no tolled link in the network (i.e., no-toll scenario), it gives 
TABLE 2: Link related parameters of the example network (min, veh/hr, $\mathrm{km}$ ).

\begin{tabular}{lcccccccccc}
\hline Link & 1 & 2 & 3 & 4 & 5 & 6 & 7 & 8 & 9 \\
\hline$t_{a}{ }^{0}$ & 7 & 9 & 9 & 12 & 3 & 9 & 5 & 13 & 5 & 9 \\
$C_{a}$ & 900 & 700 & 700 & 900 & 800 & 600 & 900 & 500 & 300 & 400 \\
$l_{a}$ & 4.0 & 4.0 & 4.0 & 7.0 & 2.0 & 4.0 & 4.0 & 8.0 & 4.0 \\
\hline Link & 11 & 12 & 13 & 14 & 15 & 16 & 17 & 18 & 19 \\
\hline$t_{a}{ }^{0}$ & 9 & 10 & 9 & 6 & 9 & 8 & 7 & 14 & 11 \\
$C_{a}$ & 700 & 700 & 600 & 700 & 700 & 700 & 300 & 700 & 700 \\
$l_{a}$ & 5.0 & 6.0 & 5.0 & 4.0 & 5.0 & 4.0 & 4.0 & 9.0 & 6.0 \\
\hline
\end{tabular}

TABLE 3: Optimal toll scheme and the corresponding link flow ( $\mathrm{min} / 0.5$, veh/hr, $\mathrm{min}$ ).

\begin{tabular}{|c|c|c|c|c|c|c|c|c|c|c|}
\hline \multicolumn{11}{|c|}{ Toll } \\
\hline Link & 1 & 2 & 3 & 4 & 5 & 6 & 7 & 8 & 9 & 10 \\
\hline$\tau_{a}^{*}$ & 1.31 & 0.29 & 0.04 & 0.52 & 0.70 & 0.15 & 0.34 & 0.02 & 0.67 & 2.10 \\
\hline$v_{a}^{*}$ & 800 & 400 & 245 & 555 & 752 & 293 & 621 & 131 & 245 & 376 \\
\hline$t_{a}^{*}$ & 7.66 & 9.14 & 9.02 & 12.26 & 3.35 & 9.08 & 5.17 & 13.01 & 5.33 & 10.05 \\
\hline Link & 11 & 12 & 13 & 14 & 15 & 16 & 17 & 18 & 19 & \\
\hline$\overline{\tau_{a}^{*}}$ & 1.95 & 0.20 & 1.23 & 0.42 & 0.18 & 0.66 & 0.00 & 0.45 & 0.81 & \\
\hline$v_{a}^{*}$ & 645 & 355 & 493 & 486 & 355 & 507 & 0 & 400 & 493 & \\
\hline$t_{a}^{*}$ & 9.97 & 10.10 & 9.62 & 6.21 & 9.09 & 8.33 & 7.00 & 14.22 & 11.41 & \\
\hline \multicolumn{11}{|c|}{ No toll } \\
\hline Link & 1 & 2 & 3 & 4 & 5 & 6 & 7 & 8 & 9 & 10 \\
\hline$v_{a}^{\prime}$ & 800 & 400 & 395 & 405 & 898 & 297 & 898 & 0 & 395 & 503 \\
\hline$t_{a}^{\prime}$ & 7.66 & 9.14 & 9.14 & 12.07 & 3.71 & 9.08 & 5.74 & 13.00 & 7.25 & 12.38 \\
\hline Link & 11 & 12 & 13 & 14 & 15 & 16 & 17 & 18 & 19 & \\
\hline$v_{a}^{\prime}$ & 795 & 205 & 497 & 205 & 205 & 503 & 0 & 400 & 497 & \\
\hline$t_{a}^{\prime}$ & 11.25 & 10.01 & 9.64 & 6.01 & 9.01 & 8.32 & 7.00 & 14.22 & 11.42 & \\
\hline
\end{tabular}

the result of deterministic user equilibrium solution without implementing road pricing scheme. In this situation, the SD-index is about 0.694, which indicates that the sustainable development level in the no-toll scenario is relatively not very high and may increase to a higher level by imposing an appropriate road toll scheme. In this example the optimal road toll scheme is $\left[\tau_{1} \tau_{2} \tau_{3} \tau_{4} \tau_{5} \tau_{6} \tau_{7} \tau_{8} \tau_{9} \tau_{10} \tau_{11} \tau_{12} \tau_{13} \tau_{14} \tau_{15} \tau_{16} \tau_{17} \tau_{18} \tau_{19}\right]=$ $\left[\begin{array}{llllllllll}1.31 & 0.29 & 0.04 & 0.52 & 0.70 & 0.15 & 0.34 & 0.02 & 0.67 & 2.10\end{array}\right.$ $\begin{array}{lllllllll}1.95 & 0.20 & 1.23 & 0.42 & 0.18 & 0.66 & 0.00 & 0.45 & 0.81] \text {, and the }\end{array}$ corresponding UE link flow is $\left[x_{1} x_{2} x_{3} x_{4} x_{5} x_{6} x_{7} x_{8} x_{9}\right.$ $\left.x_{10} x_{11} x_{12} x_{13} x_{14} x_{15} x_{16} x_{17} x_{18} x_{19}\right]=\left[\begin{array}{lll}800400245555752\end{array}\right.$ $2936211312453766453554934863555070400493]$, and with this optimal toll scheme imposed in the network, the SD-index is about 0.802 , with an increasing ratio of $15.56 \%$ compared to that in the no-toll scenario.

It can be seen from Table 4 that when the network flow pattern reaches UE, the total travel costs on all the used routes between the same OD pairs are equal to and less than the costs on all the unused routes, which is entirely consistent with the Wardrop UE principle. Besides, from Table 4 we can also find that when the optimal toll charge scheme is imposed in the network, there are 3 equilibrium routes used between OD pair $(1,3)$ under UE, while there are 2 equilibrium routes used between the same OD pairs in the no-toll scenario. In other words, it seems that the network flows under UE can be more evenly distributed throughout the whole network, and thus the traffic congestion in the road can be mitigated if the road toll scheme is properly designed. In fact, the $\mathrm{V} / \mathrm{C}$ ratios in links 5, 9, 10, and 11 are all greater than 1 under UE in the no-toll scenario, while the $\mathrm{V} / \mathrm{C}$ ratios are all smaller than 1 under UE with the optimal road toll scheme imposed on the network, which shows that the appropriate toll charge scheme can not only increase the SD-index of transportation system, but also alleviate the road congestion simultaneously.

\section{Conclusions}

In this paper, the optimal road toll design problem (TDP) from the perspective of sustainable development at networkwide level has been studied. The TDP is formulated as a bilevel programming model, and, specifically, the upper level model is to determine the optimal road toll scheme with the aim of maximizing sustainability level of transportation system and the lower level model is to depict the users' travel behaviors under a certain network toll scheme. Moreover, this paper integrates three measurable sustainability indicators of transportation system, that is, total travel time, total fuel consumption, and total vehicular emissions into a sustainable development index (SD-index), which is the final optimization objective of the bilevel programming model. Besides, the combined genetic algorithm and gradient projection 
TABLE 4: Corresponding path flow and path cost (min, veh/hr).

\begin{tabular}{|c|c|c|c|c|c|c|}
\hline \multirow{2}{*}{$(\mathrm{O}, \mathrm{D})$} & \multicolumn{3}{|c|}{ Toll } & \multicolumn{3}{|c|}{ No toll } \\
\hline & Used paths & Path flow & Path cost & Used paths & Path flow & Path cost \\
\hline$(1,2)$ & $2-18-11$ & 400 & 38.72 & $2-18-11$ & 400 & 34.62 \\
\hline \multirow{3}{*}{$(1,3)$} & $1-6-13-19$ & 293.19 & 44.78 & $1-6-13-19$ & 297.34 & 37.79 \\
\hline & $1-5-8-14-16$ & 131.27 & 44.78 & $1-5-7-10-16$ & 502.66 & 37.79 \\
\hline & $1-5-7-10-16$ & 375.54 & 44.78 & - & - & - \\
\hline \multirow{2}{*}{$(4,2)$} & $4-12-14-15$ & 354.52 & 40.28 & $4-12-14-15$ & 204.86 & 37.10 \\
\hline & $3-5-7-9-11$ & 245.48 & 40.28 & $3-5-7-9-11$ & 395.14 & 37.10 \\
\hline$(4,3)$ & 4-13-19 & 200 & 38.41 & 4-13-19 & 200 & 33.13 \\
\hline
\end{tabular}

algorithm (GA-GP) is adopted to solve the bilevel model, in which the gradient projection (GP) algorithm is mainly used to solve the lower level traffic assignment problem with a certain network toll scheme. In order to verify the proposed model and algorithm, we take the Nguyen-Dupuis network for the numerical example, and the computing results show that the model and algorithm are feasible and efficient and can be taken as a reference for the related research. Further investigations will concentrate on the toll design problem when there are multiple user groups, as well as the impacts of demand uncertainty on the optimal toll scheme.

\section{Notations}
A: The set of links in the network
R: $\quad$ The set of origins
S: The set of destinations
$\mathbf{K}^{r s}$ : The set of paths between OD pair
$(r, s), \forall r \in R, s \in S$
f: The vector of path flows, $f=\left[f_{k}^{r s}\right], \forall r \in R, s \in S$
x: $\quad$ The vector of link flows, $x=\left[x_{a}\right], \forall a \in A$
$\tau$ : $\quad$ The vector of road toll level, $\tau=\left[\tau_{a}\right], \forall a \in A$
l: $\quad$ The vector of link length, $l=\left[l_{a}\right], \forall a \in A$
U: The vector of upper bound of road toll charged on links $U=\left[U_{a}\right], \forall a \in A$
$m: \quad$ Digit number of the function value $Z(x, \tau)$, and $10^{m-1} \leqslant Z(x, \tau) \leqslant 10^{m}$
t: $\quad$ The vector of link travel times, $t=\left[t_{a}\left(x_{a}, \tau_{a}\right)\right], \forall a \in A$
$E_{a}: \quad$ The emission per vehicle on link $a$
$F_{a}: \quad 100 \mathrm{~km}$ fuel consumption per vehicle on link $a$
$\delta_{a, k}^{r s}$ : The link-path incidence, $\delta_{a, k}^{r s}=1$ if link $a$ is on the $k$ th route connecting origin $r$ and destination $s$, and $\delta_{a, k}^{r s}=0$ otherwise
$\rho$ : $\quad$ Value of time, which is assumed to be 0.5 for all travelers in this paper
$\alpha, \beta, \gamma$ : The monetary conversion factors.

\section{Conflict of Interests}

The authors declare that there is no conflict of interests regarding the publication of this paper.

\section{Acknowledgments}

The work described in this paper was supported by Grants from the National Natural Science Foundation of China (nos. 51178110, 51378119), the Graduate Innovation Project of Jiangsu Province (No. KYLX 0178), the Ph.D. Programs Foundation of Ministry of Education of China (20120092110062) and the Six Talent Peaks Program of Jiangsu Province of China (JZ-003). The authors are also grateful to the anonymous referees for providing useful comments and suggestions to improve the quality and clarity of the paper.

\section{References}

[1] H. Yan and W. H. K. Lam, "Optimal road tolls under conditions of queueing and congestion," Transportation Research Part A: Policy and Practice, vol. 30, no. 5, pp. 319-332, 1996.

[2] H. Yang and H.-J. Huang, "Principle of marginal-cost pricing: How does it work in a general road network?" Transportation Research A, vol. 32, no. 1, pp. 45-54, 1998.

[3] H. Yang, "System optimum, stochastic user equilibrium, and optimal link tolls," Transportation Science, vol. 33, no. 4, pp. 354360, 1999.

[4] Y. Yin and S. Lawphongpanich, "Internalizing emission externality on road networks," Transportation Research D, vol. 11, no. 4, pp. 292-301, 2006.

[5] L. Chen and H. Yang, "Managing congestion and emissions in road networks with tolls and rebates," Transportation Research B, vol. 46, no. 8, pp. 933-948, 2012.

[6] Z.-C. Li, W. H. K. Lam, S. C. Wong, and A. Sumalee, "Environmentally sustainable toll design for congested road networks with uncertain demand," International Journal of Sustainable Transportation, vol. 6, no. 3, pp. 127-155, 2012.

[7] G. Santos, Road Pricing: Theory and Evidence, Elsevier, Amsterdam, The Netherlands, 2004.

[8] T. D. Hau, "Congestion charging mechanisms for roads, part II-case studies," Transportmetrica, vol. 2, no. 2, pp. 117-152, 2006.

[9] X. Ban and H. X. Liu, "A link-node discrete-time dynamic second best toll pricing model with a relaxation solution algorithm," Networks and Spatial Economics, vol. 9, no. 2, pp. 243-267, 2009.

[10] Y. Lou, Y. Yin, and S. Lawphongpanich, "Robust congestion pricing under boundedly rational user equilibrium," Transportation Research Part B: Methodological, vol. 44, no. 1, pp. 1528, 2010. 
[11] H. Yang and Q. Meng, "Departure time, route choice and congestion toll in a queuing network with elastic demand," Transportation Research B, vol. 32, no. 4, pp. 247-260, 1998.

[12] E. T. Verhoef, "Second-best congestion pricing in general networks: heuristic algorithms for finding second-best optimal toll levels and toll points," Transportation Research B: Methodological, vol. 36, no. 8, pp. 707-729, 2002.

[13] X. Zhang and H. Yang, "The optimal cordon-based network congestion pricing problem," Transportation Research B, vol. 38, no. 6, pp. 517-537, 2004.

[14] Z. Liu, Q. Meng, and S. Wang, "Speed-based toll design for cordon-based congestion pricing scheme," Transportation Research Part C: Emerging Technologies, vol. 31, pp. 83-98, 2013.

[15] X. Zhang and B. van Wee, "Enhancing transportation network capacity by congestion pricing with simultaneous toll location and toll level optimization," Engineering Optimization, vol. 44, no. 4, pp. 477-488, 2012.

[16] H. Li, M. C. J. Bliemer, and P. H. L. Bovy, "Network reliabilitybased optimal toll design," Journal of Advanced Transportation, vol. 42, no. 3, pp. 311-332, 2008.

[17] H. Yang and H. J. Huang, Mathematical and Economic Theory of Road Pricing, Elsevier, Amsterdam, The Netherlands, 2005.

[18] A. de Palma and R. Lindsey, "Traffic congestion pricing methodologies and technologies," Transportation Research C, vol. 19, no. 6, pp. 1377-1399, 2011.

[19] C. E. Wallace, K. G. Courage, M. A. Hadi, and A. C. Gan, TRANSYT-7F User's Guide, University of Florida, Gainesville, Fla, USA, 1998.

[20] L. Xu and G.-Z. Cheng, "Setting minimum vehicle speed limit on freeway based on speed scattering and economic speed," Journal of Jilin University, vol. 40, no. 3, pp. 661-665, 2010.

[21] D. Goldberg, Genetic Algorithms in Search, Optimization and Machine Learning, Addison-Wesley, Reading, Mass, USA, 1989.

[22] T. Gen and R. Cheng, Genetic Algorithms and Engineering Design, New York, NY, USA, John Wiley \& Sons, 1997.

[23] X.-D. Xu and L. Cheng, "Bilevel programming model and hybrid solution algorithm for the configuration of one-way streets," System Engineering Theory and Practice, vol. 29, no. 10, pp. 180-187, 2009.

[24] T. Xu, H. Wei, and G. Hu, "Study on continuous network design problem using simulated annealing and genetic algorithm," Expert Systems with Applications, vol. 36, no. 2, pp. 1322-1328, 2009.

[25] S. Nguyen and C. Dupuis, "An efficient method for computing traffic equilibria in networks with asymmetric transportation costs," Transportation Science, vol. 18, no. 2, pp. 185-202, 1984. 


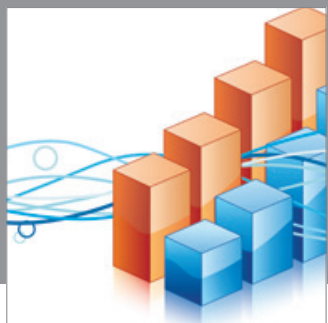

Advances in

Operations Research

mansans

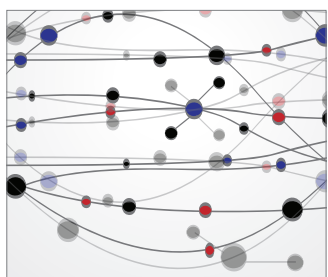

The Scientific World Journal
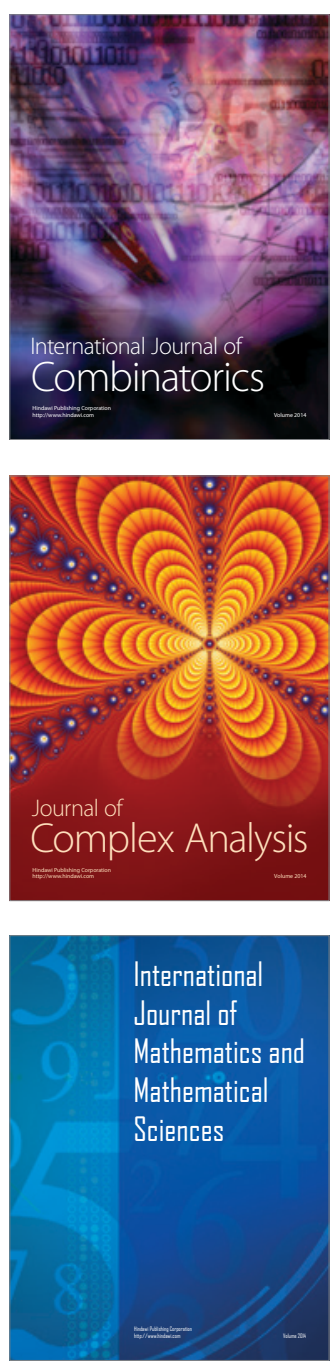
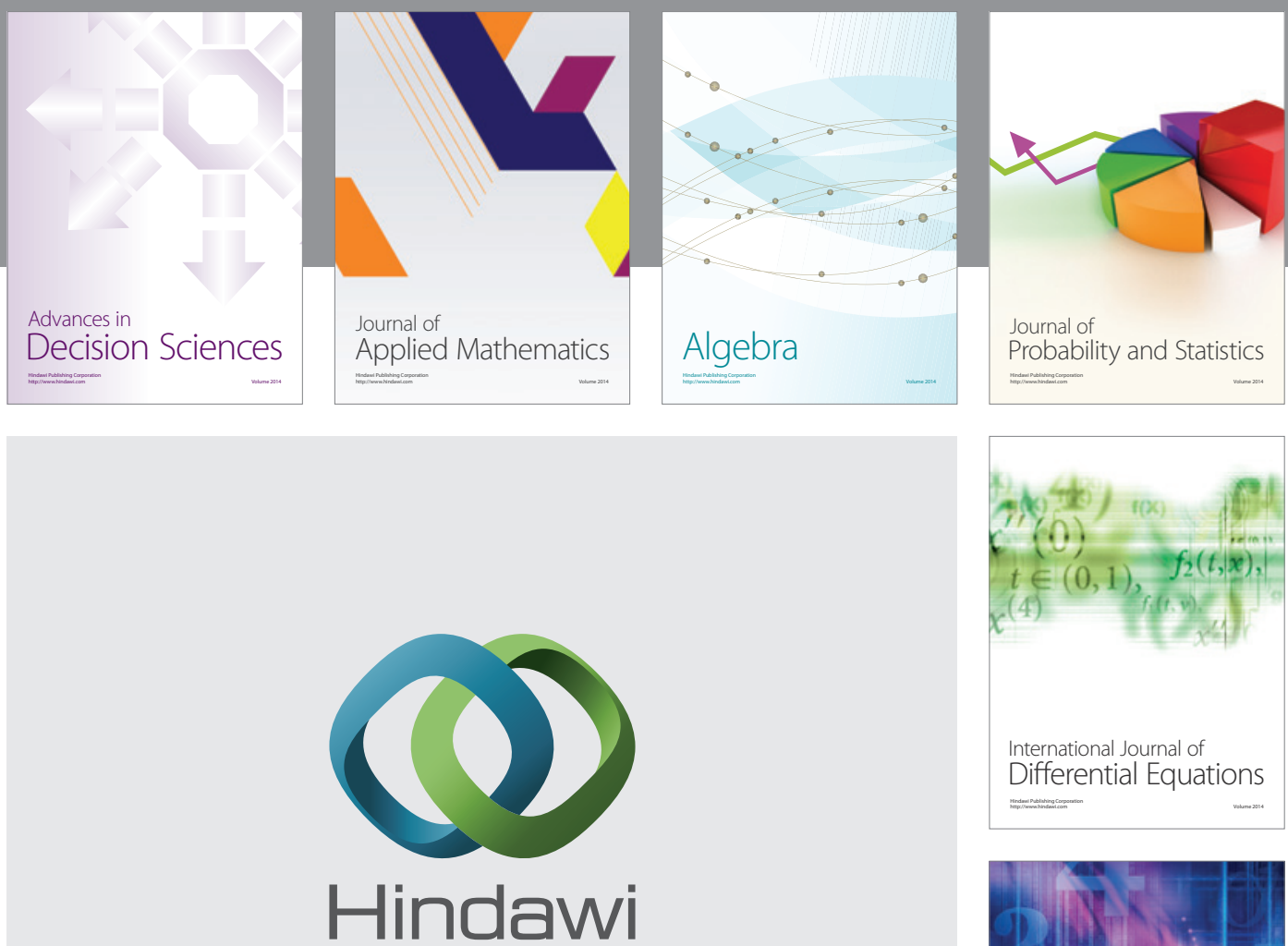

Submit your manuscripts at http://www.hindawi.com
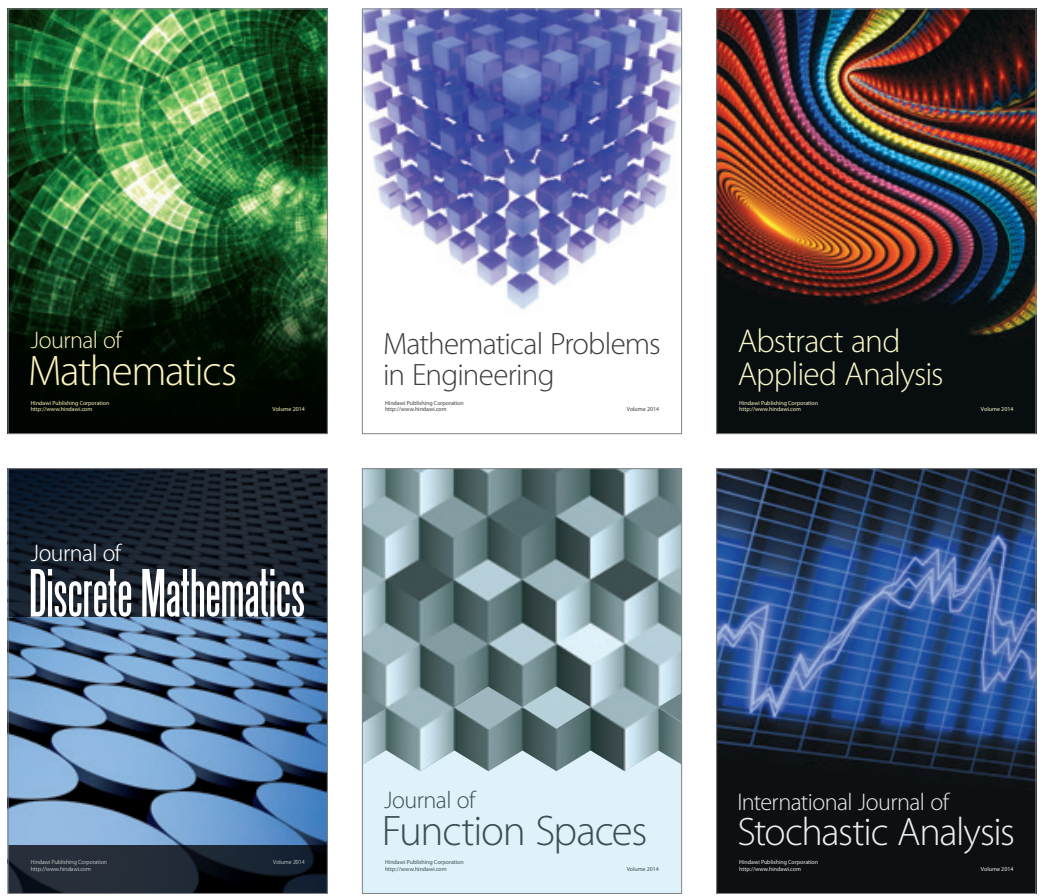

Journal of

Function Spaces

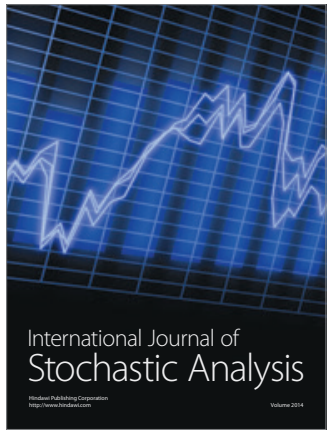

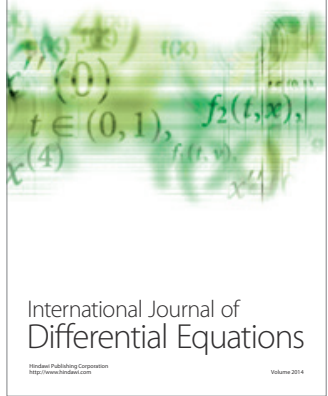
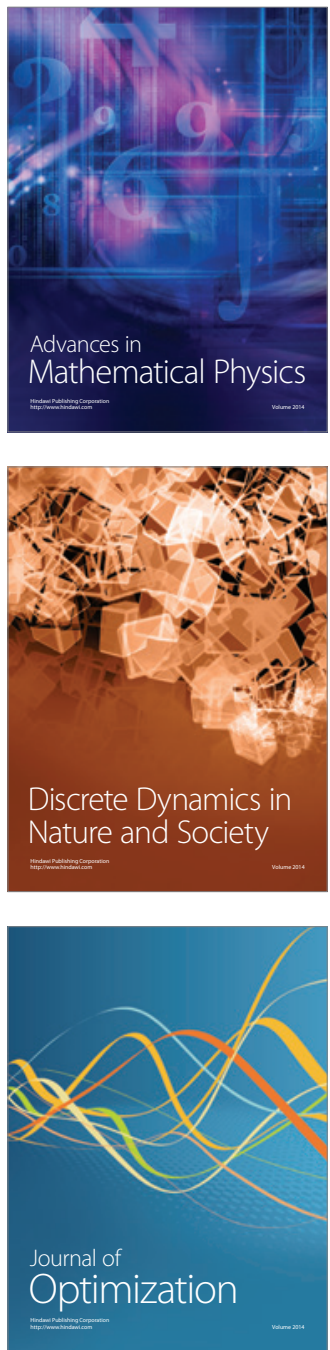